\title{
Aspectos psicológicos y Doppler-Duplex dinámico en disfunción eréctil
}

\author{
M.A. Fernández-Gil*, L. Martínez-Piñeiro**, M. Martí***, F.J. Vaz Leal****, \\ J.A. Guisado Macías***** \\ *Servicio de Radiología. Hospital Infanta Cristina. Badajoz. **Servicio de Urología. Hospital Universitario \\ La Paz. Madrid. ***Servicio de Radiología. Hospital Universitario La Paz. Madrid. \\ ****Departamento de Psiquiatría. Universidad de Extremadura. ${ }^{* * * * * S e r v i c i o ~ d e ~ P s i q u i a t r i ́ a . ~}$ \\ Hospital Infanta Cristina. Badajoz.
}

Actas Urol Esp 2005; 29 (8): 884-889

\begin{abstract}
RESUMEN
ASPECTOS PSICOLÓGICOS Y DOPPLER-DUPLEX DINÁMICO EN DISFUNCIÓN ERÉCTIL

Objetivo: Relacionar los hallazgos de la ecografía Doppler-Duplex color tras la inyección intracavernosa de prostaglandina con los aspectos psicológicos en pacientes con disfunción eréctil (DE).

Material y método: Se estudiaron, de forma prospectiva, 42 pacientes con DE mediante ecografía Doppler-Duplex color tras la inyección de 20 microgramos de $\mathrm{PGE}_{1}$ intracavernosa. Se evaluaron los patrones morfodinámicos y de flujo. Se graduó visualmente la tumescencia peneana en pobre, moderada o buena (I, II, III). Todos los pacientes completaron el Índice Internacional de Función Eréctil (IIFE). Para el estudio psicológico de los pacientes se utilizó el examen Internacional de los Trastornos de la Personalidad (IPDE, módulo CIE-10) y Symptom Checlist 90-Revised (SCL-90R).

Resultados: 29 pacientes (69.05\%) mostraron una respuesta normal en el estudio ecográfico (velocidad picosistólica >30 $\mathrm{cm} / \mathrm{s}$; velocidad telediastólica negativa o < de $5 \mathrm{~cm} / \mathrm{s}$ y tumescencia peneana grado III) y fueron clasificados como pacientes con $\mathrm{DE}$ no vascular y probablemente psicógena. El resto de los pacientes que no cumplían estos criterios se clasificaron dentro del grupo de DE de etiología vascular. Encontramos diferencias significativas entre los grupos en el deseo sexual, grado de tumescencia y personalidad paranoide. Los rasgos de personalidad más frecuentes fueron paranoide y esquizoide (personalidades excéntricas) y anancástico-ansioso (personalidades temerosas). La somatización y síntomas obsesivo-compulsivos han sido los más destacados. Existe relación entre la personalidad paranoide y la exploración doppler normal.

Conclusión: La existencia de ciertos aspectos psicopatológicos y rasgos disfuncionales de personalidad acompaña a la DE tanto orgánica como psicógena, siendo lo más frecuente una etiología mixta. La ecografía doppler es un método eficaz en el estudio de los pacientes con DE. Una exploración doppler normal indica una adecuada respuesta vascular en el mecanismo de la erección y se relaciona con ciertas alteraciones psicológicas y rasgos de personalidad. El análisis de los aspectos psicológicos de estos pacientes permite una orientación terapéutica más adecuada.
\end{abstract}

Palabras clave: Disfunción eréctil, Ecografia Doppler, Trastornos de la Personalidad, Trastornos Mentales.

\section{ABSTRACT}

PSYCHOLOGICAL FEATURES AND DOPPLER-DUPLEX IN ERECTILE DISFUNCTION

Objectives: To establish a relationship between Doppler-Duplex colour ultrasound after prostaglandin intracorporeal injection and psychological features in patients suffering from erectile dysfunction.

Methods: Forty two patients with erectile dysfunction were prospectively evaluated with Doppler-Duplex colour ultrasonography after intracavernosal injection of 20 micrograms of $E_{1}$ prostaglandin. Dynamic vascular pattern were analyzed and penile tumescence were graded in poor, moderate or good (I, II, III). All of them completed the International Index of Erectile Dysfunction, International Exam of Personality Traits (IPDE) and the Symptom Checklist (SCL-90).

Results: 29 patients $(69.05 \%)$ showed a normal ultrasonography response (Peak Systolic Velocity $>30 \mathrm{~cm} / \mathrm{s}$; Telediastolic velocity negative or less than $5 \mathrm{~cm} / \mathrm{s}$ and penile tumescence grade III) and were classified as good responders with probably psychogenic erectile dysfunction. Patients who did not show these values were classified in the group of erectile dysfunction of vascular origin. We found significative differences between the two groups in sex desire, tumescence and paranoid personality. The more frequent personality features were paranoid and squizoid (excentric) and ananchastic and anxious (fearful ones). Somatization and obsessive-compulsive symptoms have been outlined over the rest. We have found that paranoid personality is sixteen times more frequent in patients with normal ultrasonography.

Conclusions: Psychological features and dysfunctional personality traits accompany psychogenic and organic erectile dysfunction, thus it is thought that mixed aetiology coexist in this patients. Doppler-Dupplex Colour ultrasound is an useful method to exclude organic vascular factors. On de basis of our article, is more common to find psychological discomfort and dysfunctional personality traits in patients with normal ultrasound, which may help in their diagnosis and treatment.

Keywords: Erectile Dysfunction, Doppler Sonography, Personality disorders, Psychopathology, Mental Disorders. 
$\mathrm{S}_{\mathrm{p}}^{\mathrm{e}}$ entiende por disfunción eréctil (DE) la incapacidad para mantener o conseguir la suficiente rigidez del pene para realizar una relación sexual satisfactoria ${ }^{1}$.

En España la prevalencia de DE estimada es aproximadamente $18,9 \%$ entre los 25 y 70 años, existiendo relación con algunos factores de riesgo (cardiovasculares, ingesta de drogas, enfermedad prostática, alergia, enfermedades reumáticas, tabaco, alcohol, e incluso la edad) ${ }^{2}$.

Clásicamente se ha considerado la causa psicógena como la principal etiología de la DE aunque estudios recientes han puesto de manifiesto que el $78 \%$ de los pacientes mostraban un origen orgánico con o sin componentes psicógenos ${ }^{3}$.

Un alto porcentaje de casos es producto de una causa orgánica arterial (fibrosis de cuerpos cavernosos, insuficiencia arterial o incompetencia venosa) para lo cual se requiere un estudio de la vascularización del pene que puede llevarse a cabo con la ecografía doppler-duplex color ${ }^{4}$. En los pacientes con una respuesta normal (velocidad picosistólica mayor de $30 \mathrm{~cm} / \mathrm{s}$ acompañada de tumescencia peneana grado II-III) en la ecografía se puede excluir una causa orgánica vascular, siendo más probable la etiología psicógena. Esta técnica, sin embargo, no excluye la existencia de alteraciones neurológicas $u$ hormonales.

En la actualidad se cree que existe un continuo entre ambas etiologías, encontrándose una causa mixta en la mayoría de los casos: la incapacidad para lograr la erección produce en el individuo ansiedad, pérdida de la autoestima, sentimientos de culpabilidad, miedo o depresión. Los sujetos con niveles de ansiedad elevados y trastornos de la personalidad (individuos con patrones de comportamiento anormales), muestran elementos de inestabilidad afectiva y alta reactividad emocional que pueden desencadenar el miedo y la ansiedad anticipatoria que preceden a la disfunción eréctil psicógena ${ }^{5}$.

El objetivo del presente estudio fue relacionar los hallazgos de la ecografia doppler con los aspectos psicológicos en los pacientes con disfunción eréctil y aportar datos sobre las características psicológicas de los enfermos que demandan tratamiento por esta causa.

\section{MATERIAL Y METODO}

Se estudiaron 42 pacientes varones que consultaron en el servicio de Urología del Hospital Universitario "La Paz" de Madrid por disfunción eréctil. Los pacientes con impotencia neurogénica (en base a la historia clínica y a la exploración neurológica básica) no fueron incluidos en el estudio. Todos fueron evaluados por un urólogo realizándose historia clínica y examen físico completos, así como hemograma y bioquímica sanguínea incluyendo niveles hormonales (testosterona, prolactina).

En la consulta, todos los pacientes completaron el Índice Internacional de Función Eréctil $(\mathrm{IIFE})^{6}$ que es una escala auto administrada y multidimensional que mediante quince preguntas evalúa aspectos relevantes de la función sexual masculina (función eréctil, función orgásmica, deseo sexual, satisfacción sexual y satisfacción global).

Para el estudio de la personalidad se utilizó el Examen Internacional de los Trastornos de la Personalidad (IPDE, módulo CIE-10) ${ }^{7}$ que consiste en una entrevista clínica semiestructurada diseñada para evaluar los trastornos de la personalidad. Consta de 67 preguntas donde se agrupan los trastornos de la personalidad paranoide (desconfiados), esquizoide (fríos raros), disocial (no aceptan normas sociales), inestabilidad emocional (tipo impulsivo y tipo límite), histriónico (que llaman la atención), anancástico (obsesivo), ansioso (temeroso) y no especificado. Esta escala evalúa si existe algún perfil de conducta anómalo en la vida diaria.

La valoración psicopatológica se realizó con la escala Sympton Checklist (SCL-90 R) ${ }^{8}$. Es una auto-escala que se compone de 90 apartados que evalúan la sintomatología psiquiátrica en pacientes ambulatorios. Cada ítem se gradúa en cinco puntos que oscilan entre "nada en absoluto" hasta "extremo". La SCL-90 aporta información sobre 9 subescalas (somatización, obsesivo-compulsivo, sensitividad interpersonal, depresión, ansiedad, angustia/hostilidad, ansiedad fóbica, ideación paranoide y psicoticismo) así como un índice medio, que es la media aritmética de la puntuación de los 90 apartados (GSI, Índice de Severidad Global). Con esta escala evaluamos los síntomas de malestar psicológico que presenta el 
paciente en la semana previa a la valoración urológica. Se consideran patológicos los síntomas de las subescalas que cuyo GSI es mayor de 1 .

Todos los pacientes fueron remitidos al servicio de radiología para realizar ecografía abdominal y estudio ecográfico peneano en modo $\mathrm{B}$ y Duplex-Doppler color basal y tras la administración de intracavernosa de $20 \mathrm{mcg}$. de prostaglandina $\mathrm{E}_{1}$ tomando medidas de forma dinámica a los $5,10,15$ y 20 minutos, con registro de las distintas fases espectrales y de flujo.

Se evaluó la velocidad pico sistólica, la velocidad telediastólica y la inversión de la diástole en la fase final. Asimismo se midió el volumen de los cuerpos cavernosos y el calibre de las arterias cavernosas antes y después de la administración de prostaglandina.

Graduamos la tumescencia peneana tras la administración del agente vasodilatador clasificando la misma en pobre, moderada o buena (grados I, II y III).

Consideramos un patrón hemodinámico normal cuando la velocidad pico-sistólica es mayor de $30 \mathrm{~cm} / \mathrm{seg}$, la velocidad telediastólica es menor de $5 \mathrm{~cm} / \mathrm{s}$ o negativa, la tumescencia peneana es buena (grado III) y la erección dura, al menos, cinco minutos.

Apoyamos el diagnóstico de causa orgánica vascular arterial cuando no se alcanza la velocidad picosistólica de $30 \mathrm{~cm} / \mathrm{s}$ asociándose o no a una causa venosa en función de la velocidad telediastólica (mayor de $5 \mathrm{~cm} / \mathrm{s}$ ) e índice de resistencia (IR) (el valor normal es 1 pero se acepta que valores de IR por encima de 0,75 descartarian fuga venosa).

En base a estos resultados hemodinámicos, clasificamos a los pacientes como causa no vascular (probablemente psicógena) y causa vascular.

Realizamos estudio descriptivo de los hallazgos ecográficos y psicológicos.

El análisis estadístico se realizó mediante: Chi-cuadrado para el estudio de las variables cualitativas, T-test para el estudio de las variables cuantitativas y el análisis de regresión logística para ver qué tipo de personalidad se relaciona con la existencia de una exploración doppler normal. Se calculó la odds ratio y el intervalo de confianza (IC) del 95\%, estableciéndose la significación estadística en $\mathrm{p}<0,05$.
Se utilizó el programa estadístico SPSS v. 10.0 para windows.

\section{RESULTADOS}

La muestra consta de 42 pacientes con edad media de 41,04 $\pm 8,96$ años. Veintiseis pacientes $(61,9 \%)$ eran casados y $16(38,1 \%)$ solteros. Treinta y ocho $(90,5 \%)$ pacientes desarrollaban una actividad laboral estable. Veintidos $(52,6 \%)$ eran fumadores. La mayoría desarrollaban erecciones matutinas y con la masturbación en un porcentaje de 61,9 (26 pacientes) y 57,14 (24 pacientes), respectivamente. La edad media de inicio de los síntomas fue de 37,23 \pm 9,26 años.

Tras la administración intracavernosa de prostaglandina $\mathrm{E}_{1}, 8$ pacientes, $(19,04 \%)$ mostraron una tumescencia peneana grado I, 9 $(21,42 \%)$ grado II y $25(59,52 \%)$ grado III.

Veintinueve pacientes $(69,04 \%)$ fueron clasificados en el grupo "disfunción eréctil de causa no vascular". Trece pacientes $(30,95 \%)$ fueron clasificados dentro del grupo "disfunción eréctil de causa orgánica, posiblemente vascular” (Tabla 1).

En el estudio de personalidad (IPDE), los rasgos disfuncionales de carácter más frecuentes en nuestra población fueron paranoide, anancástico, esquizoide y ansioso (Fig. 1).

En la escala SCL-90 destacan por encima del índice de severidad global los síntomas de somatización y obsesivo-compulsivo (Fig. 2).

La suma de las puntuaciones de los diferentes dominios del cuestionario IIFE fue mayor en los pacientes con exploración doppler normal $(26,16)$ frente a aquellos que mostraron una exploración doppler patológica $(21,40)$.

Del mismo modo, la puntuación media en cada dominio de la escala IIFE (función eréctil, satisfacción en las relaciones, orgasmo, deseo sexual y satisfacción general) fue mayor en el grupo de pacientes con respuesta ecográfica normal tras la inyección de PGE1 intracavernosa $(7,5-3,3-5,0-7,0$ y 3,3$)$ que en aquellos con respuesta no adecuada (5,6-2,8-4,0-3,0 y 6,0)

Se encontraron diferencias significativas entre los dos grupos de pacientes en los siguientes items: dominio deseo sexual de la escala IIFE, grosor de los cuerpos cavernosos medido con ecografía modo B, grado de tumescencia peneana y personalidad paranoide (Tablas 2 y 3 ). 
Tabla 1

\begin{tabular}{|c|c|c|c|c|c|}
\hline & & & $\begin{array}{l}\text { Velociadad } \\
\text { media (VPS) } \\
\text { Cm/s }\end{array}$ & $\begin{array}{l}\text { Velocidad } \\
\text { media } \\
\text { (VTD) Cm/ s }\end{array}$ & № pacientes \\
\hline \multicolumn{3}{|c|}{$\begin{array}{l}\text { No orgánica } \\
\text { Psicógena }\end{array}$} & $45,33 \pm 10.04$ & $<0$ & 29 \\
\hline \multirow[t]{3}{*}{$\begin{array}{l}\text { Orgánica } \\
\text { Vascular }\end{array}$} & $\begin{array}{l}\text { Venosa } \\
\text { E. venooclusiva }\end{array}$ & & $45,95 \pm 12.52$ & $11,15 \pm 4,80$ & 4 \\
\hline & $\begin{array}{l}\text { Arterial } \\
\text { Insuf. arterial }\end{array}$ & $\begin{array}{l}\text { Pura o } \\
\text { con función } \\
\text { venosa buena }\end{array}$ & $19,52 \pm 8.41$ & $\begin{array}{l}3,78 \pm 2,34 \\
<0\end{array}$ & $\begin{array}{l}4 \\
2\end{array}$ \\
\hline & Bordeline & & $31 \pm 2,73$ & $\begin{array}{l}>0 \\
<0\end{array}$ & 3 \\
\hline Total & & & & & 42 \\
\hline
\end{tabular}

Clasificación de la disfunción y parámetros ecográficos (velocidades picosistólicas -VPS- y telediastólicas -VTD-) que los definen

El análisis de regresión logística presentó una relación entre el diagnóstico no vascular, posiblemente psicógeno (como variable dependiente) y la personalidad paranoide (odds ratio $=16,00$; IC del 95\%: de 1,31-19,62; p< 0,01).

\section{DISCUSIÓN}

La erección se lleva a cabo, entre otros, mediante mecanismos hemodinámicos que implican el incremento del flujo arterial cavernoso, la relajación del músculo liso de los cuerpos cavernosos y la activación del mecanismo veno-oclusivo.
Por ello, los estudios hemodinámicos son importantes para evaluar a los pacientes con disfunción eréctil (DE) ${ }^{9}$. La ecografía doppler-duplex color con inyección de prostaglandina intracavernosa es una técnica con escasos efectos secundarios que evalúa la circulación peneana y permite establecer o descartar la existencia de una causa orgánica vascu$\operatorname{lar}^{10}$. En nuestro estudio el $69,05 \%$ de los pacientes tenían una ecografía doppler normal, lo que permitía excluir la existencia de una causa orgánica vascular siendo más probable la etiología psicógena. En este sentido, discrepamos con Slob y colaboradores ${ }^{11}$, ya que consideramos que la ecografía doppler-duplex color realizada de forma dinámica y por un ecografista experto es una herramienta útil en el despistaje de la etiología de la disfunción eréctil y ayuda a la orientación terapéutica del problema. La respuesta normal en la ecografía de nuestros pacientes se correlaciona con el porcentaje de los mismos que presentaban erecciones nocturnas y matutinas y niveles hormonales normales.

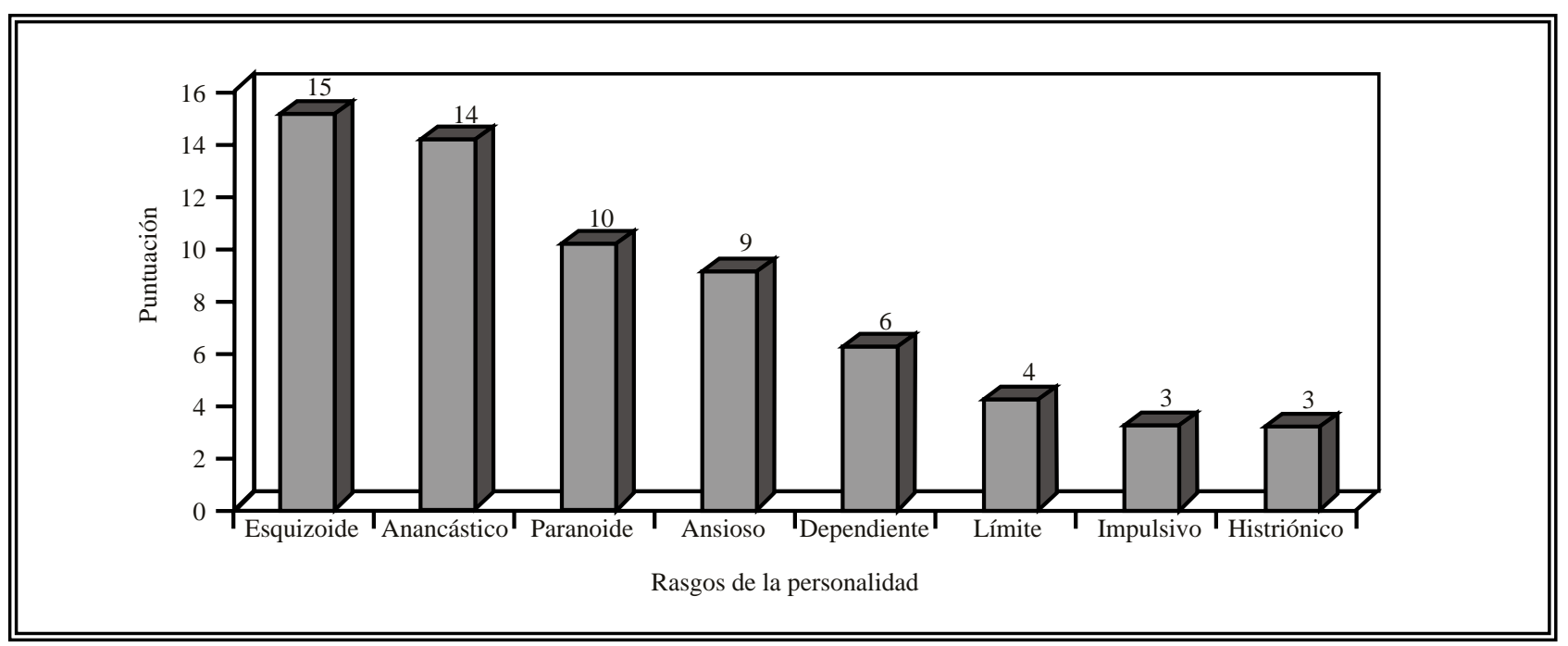

FIGURA 1. Examen Internacional de los Trastornos de la Personalidad (IPDE, módulo CIE-10). En el eje " $x$ " se representan los rasgos de personalidad y en el eje " $y$ " la puntuación obtenida en términos absolutos. Los pacientes mostraron puntuación más alta en los rasgos de personalidad esquizoide, anascástico, paranoide y ansioso. 


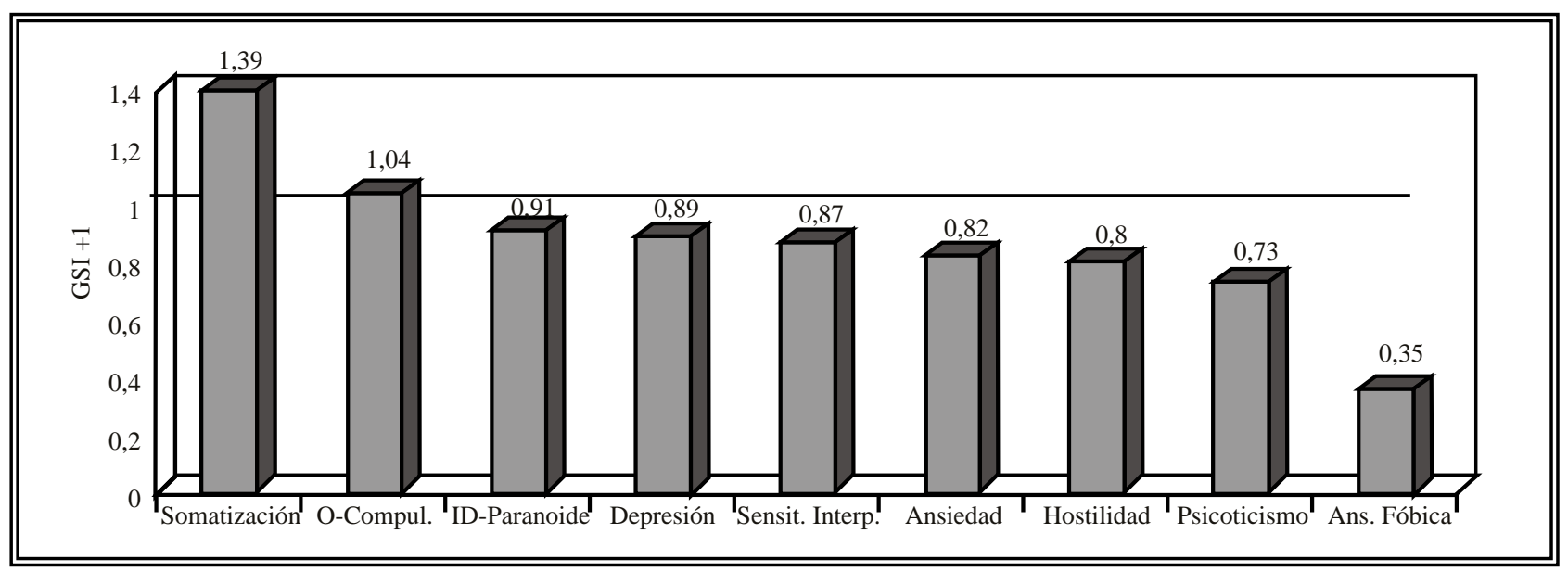

FIGURA 2. Sintomas de malestar psicológico (abscisas). Symptom Checklist (SCL-90R). GSI (Índice de Severidad Global). Los valores de GSI (ordenadas) que se encuentran por encima de 1 son considerados patológicos.

Tabla 2

Variables cualitativas

\begin{tabular}{lcccc}
\hline & $\begin{array}{c}\text { No orgánico } \\
\mathbf{n}\end{array}$ & $\begin{array}{c}\text { Organico } \\
\mathbf{n}\end{array}$ & $\mathbf{X}^{2}$ & $\mathbf{p}$ \\
\hline $\begin{array}{l}\text { Tumescencia } \\
\text { Grado I }\end{array}$ & 0 & 8 & & \\
Grado II-III & 28 & 6 & 0,38 & 0,01 \\
$\begin{array}{l}\text { IPDE paranoide } \\
\text { Positivo }\end{array}$ & 20 & 2 & & \\
Negativo & 8 & 12 & 1,00 & 0,05 \\
\hline
\end{tabular}

Rasgos descriptivos de nuestra muestra. Asociación del grado de tumescencia y la personalidad paranoide con la existencia de alteraciones orgánicas y no orgánicas.

Tabla 3

Variables cuantitativas

\begin{tabular}{lcccc}
\hline & $\begin{array}{c}\text { No orgánico } \\
\text { media } \pm \\
\text { desviación } \\
\text { estándar }\end{array}$ & $\begin{array}{c}\text { Orgánico } \\
\text { media } \pm \\
\text { desviación } \\
\text { estándar }\end{array}$ & t & p \\
\hline Grosor CC (mm) & & & & \\
Antes & $10,88 \pm 2,28$ & $8,03 \pm 1,19$ & 2,746 & 0,001 \\
Después & $21,61 \pm 2,85$ & $15,87 \pm 4,11$ & 3,171 & 0,001 \\
Deseo sexual & $7,00 \pm 0,89$ & $3,00 \pm 1,41$ & 0,36 & 0,001 \\
\hline
\end{tabular}

Rasgos descriptivos de nuestra muestra. Asociación entre el grosor de los cuerpos cavernosos y el deseo sexual con la existencia de alteraciones orgánicas y no orgánicas.

Podemos simplificar la etiología de la DE dividiendo sus causas en psicógena, orgánica o mixta, si bien, parece que el mayor porcentaje de los casos se encuentran en el último grupo ya que parece existir un continuo entre lo psicógeno y lo orgánico de manera que los factores orgánicos interactúan con los puramente psicógenos y los disparan además de que cualquier enfermedad orgánica se acompaña de ciertos aspectos psicológicos ${ }^{5}$.
La estructura de la personalidad es un factor importante que influye en el desarrollo y mantenimiento de la disfunción eréctil, de manera que los sujetos con mayor inestabilidad emocional experimentarían niveles de ansiedad elevados que les producirian interferencia y bloqueo de los mecanismos responsables de la excitación y el orgasmo ${ }^{12}$. Nuestros pacientes, independientemente de la etiología de la disfunción eréctil, los rasgos de personalidad más frecuentes que presentaron fueron: paranoide (desconfianza, suspicacia), esquizoide (frialdad, rareza), anancástico (rigidez, obsesión) y ansioso (temerosos y ansiosos). Estos rasgos caracterizan a los pacientes como sujetos introvertidos, mal socializados y desajustados emocionalmente; si bien mientras las personalidades paranoide y esquizoide hablan de personas extrañas e independientes, los anancásticos y ansiosos corresponden a sujetos temerosos y dependientes ${ }^{13}$. Estos datos muestran dos aspectos de nuestra población: por un lado, al ser pacientes con más problemas a nivel emocional y malestar psicológico demandarian más los servicios ambulatorios provocando un sesgo en nuestra muestra; y por otro, el desajuste emocional podría ser un factor directo que causa y mantiene la disfunción eréctil en los mismos.

Mediante el análisis de regresión logística observamos que los pacientes con una exploración doppler normal, en los que excluimos una alteración orgánica vascular, la personalidad paranoide es aproximadamente dieciséis veces más frecuente que los que muestran patrones ecográficos altera- 
dos, lo que demuestra, de nuevo, que en nuestros pacientes el componente emocional y la normalidad ecográfica estaban muy unidos.

Los sintomas obsesivo-compulsivo y somatización han puntuado por encima del Índice de Severidad Global en la escala SCL-90, lo que demuestra la existencia de malestar psicológico en individuos rumiadores con abundantes quejas físicas, neuroticismo y ansiedad que conllevaría a la inhibición de la respuesta sexual. Estos individuos encajan en el modelo propuesto por Barlow $^{14}$ en el que los sujetos con rumiaciones (obsesivos) desarrollan conductas evitativas a partir de la disminución de la autoestima y autoconfianza al subestimar el nivel de excitación alcanzado y a la pérdida del control sobre la respuesta eréctil lo que conlleva una gran ansiedad que es el factor principal de desencadenamiento y mantenimiento de la $\mathrm{DE}^{5,15}$.

Los valores medios obtenidos en los dominios de la escala IIFE para los pacientes que responden adecuadamente en el estudio doppler son mayores que los de aquellos con respuesta insuficiente en la ecografía, aunque sólo existen diferencias estadísticamente significativas entre los dos grupos en la subescala "deseo sexual". Si consideramos el dominio "función eréctil" ambos grupos muestran una disfunción eréctil severa ${ }^{16}$. La existencia de puntuaciones bajas en esta escala concuerda con los resultados de Blander ${ }^{17}$ : La escala IIFE es un instrumento válido para evaluar la satisfacción sexual masculina y la severidad de la disfunción, si bien no distingue entre las etiologías. Además, es un instrumento subjetivo que refleja la percepción que el paciente tiene de su disfunción eréctil, por lo que aunque el mecanismo eréctil sea normal en el estudio ecográfico dinámico, el paciente se considera impotente, lo que queda reflejado en el resultado del cuestionario.

\section{CONCLUSIÓN}

Los pacientes con disfunción eréctil, independientemente de la causa, muestran aspectos psicológicos especiales. La ecografía doppler es un método eficaz en el estudio de los pacientes con DE. Una exploración doppler normal indica una adecuada respuesta vascular en el mecanismo de la erección relacionándose con ciertos rasgos o alteraciones de la personalidad. En este grupo de pacientes la personalidad paranoide es la más frecuentemente encontrada. Proponemos, desde nuestra experiencia, la protocolización y evaluación psicológica-psiquiátrica de todos los pacientes que demandan asistencia por DE ya que el análisis de los aspectos psicológicos de estos pacientes permite una orientación terapéutica más adecuada.

\section{REFERENCIAS}

1. NIH Consensus Conference: Impotence. NIH Development Panel on Impotence. JAMA 1993; 270:83-90.

2. Martín-Morales A, Sánchez-Cruz JJ, Saenz de Tejada I et al. Prevalence and independent risk factors for erectile dysfunction in Spain: Results of the Epidemiologia de la Disfunción Eréctil Masculina Study. The J. Urol. 2001; 166:569-575.

3. Feldman HA, Goldstein I, Hatzichristou DG et al. Impotence and its medical and psychological correlates: results of the Massachussets Male Aging Study. J. Urol. 1994;151(1):54-61.

4. Hattery RR, King BF, Lewis RW et al. Vasculogenic impotence. Duplex and color Doppler Imaging. Radiol Clin North Am. 1991;29(3):629-645.

5. Farre JM, Laceras MG. Disfunción eréctil psicógena y mixta: Estudio de seguimiento terapéutico. Actas Esp Psiquiatr. 2002; 30:38-45.

6. Rosen RC, Riley A, Wagner G, et al. The international index of erectile function (IIEF): a multidimensional scale for assessment of erectile dysfunction. Urology.1997;49(6): 822-830.

7. Examen Internacional de los Trastornos de la Personalidad (IPDE) Módulo CIE-10. Ginebra: Organización Mundial de la Salud,1996.

8. Derogatis LR. SCL-90 R version. Manual I. Baltimore: John Hopkins University School of Medicine, 1977.

9. Chiou RK, Pomeroy BD, Chen WS et al. Hemodynamic patterns of pharmacologically induced erection: evaluation by color Doppler sonography”. J. Urol.1998;159:109-112.

10. Vidal Moreno JF, Moreno Pardo B, Jimenez Cruz JF. Disfunción eréctil de causa arterial. Estudio vascular del pene con Eco-Doppler. Actas Urol Esp.1996;20(5):421-427.

11. Slob AK, Cornelissen S, Dohle GR et al. The limited practical value of color doppler sonography in the differential diagnosis of men with erectile dysfunction. Int J Impot Res. 2002;14:201-203.

12. Kockott G, Feil W, Ferstl R et al. Symptomatology and psychological aspects of male sexual inadecuacy : Results of an experimental study. Arch Sex Behav. 1980;9(6):457-475.

13. Am. Psych. Association: Manual de diagnóstico y estadística de los trastornos mentales. 4 $4^{\mathrm{a}}$ edición. Masson: Barcelona 1995.

14. Beck JG, Barlow DH. The effects of anxiety and attentional focus on sexual responding II. Cognitive and affective patterns in erectile dysfunction. Behav Res Ther. 1986;24(1):19-26.

15. Farré JM, Lasheras MG. Factores psicógenos en la disfunción eréctil. Actas Esp Psiquiatr. 1999;27 (90):16-21.

16. Cappelleri JC, Rosen RC, Smith MD, Mishra A, Osterloh IH. Diagnostic evaluation of the erectile function domain of the International Index of Erectile Function. Urology. 1999 Aug; 54 (2): 346-351.

17. Blander DS, Sanchez-Ortiz RF, Broderick GA. Sex inventories: can questionnaires replace erectile dysfunction testing?. Urology. 1999; 54(4):719-723.

Dr. MaA. Fernández Gil

Jesús Rincón Jiménez, 82 - 5ํD

06010 Badajoz

e-mail: mafdezgil@telefonica.net

(Trabajo recibido el 23 de febrero 2005) 